\title{
ON STEIN FILLINGS OF CONTACT TORUS BUNDLES
}

\author{
MARCO GOLLA AND PAOLO LISCA
}

\begin{abstract}
We consider a large family $\mathcal{F}$ of torus bundles over the circle, and we use recent work of Li-Mak to construct, on each $Y \in \mathcal{F}$, a Stein fillable contact structure $\xi_{Y}$. We prove that (i) each Stein filling of $\left(Y, \xi_{Y}\right)$ has vanishing first Chern class and first Betti number, (ii) if $Y \in \mathcal{F}$ is elliptic then all Stein fillings of $\left(Y, \xi_{Y}\right)$ are pairwise diffeomorphic and (iii) if $Y \in \mathcal{F}$ is parabolic or hyperbolic then all Stein fillings of $\left(Y, \xi_{Y}\right)$ share the same Betti numbers and fall into finitely many diffeomorphism classes. Moreover, for infinitely many hyperbolic torus bundles $Y \in \mathcal{F}$ we exhibit non-homotopy equivalent Stein fillings of $\left(Y, \xi_{Y}\right)$.
\end{abstract}

\section{INTRODUCTION}

The diffeomorphism classification of symplectic fillings has been previously considered by several authors. For the standard definitions on symplectic structures, contact structures and their symplectic fillings we refer the reader to [6, 18]. The first classification result for symplectic fillings is due to Eliashberg [3], who proved that a symplectic filling of the standard contact $S^{3}$ is diffeomorphic to a blowup of $B^{4}$. McDuff [16] extended Eliashberg's result to the lens spaces $L(p, 1)$ endowed with their standard contact structures. Ohta and Ono [20,21] determined the diffeomorphism types of symplectic fillings of links of simple elliptic and simple singularities endowed with their natural contact structures. Stein fillings up to diffeomorphisms were classified by the second author [12] for all lens spaces with their standard contact structures, by Plamenevskaya-Van Horn-Morris [22] on $L(p, 1)$ with other contact structures and by Starkston [23] for certain contact Seifert fibered 3-manifolds. In this paper we study Stein and symplectic fillings of infinitely many contact torus bundles over the circle.

Below we define a large family $\mathcal{F}$ of closed, oriented torus bundles over $S^{1}$, and in Section 2 we use recent work of Li-Mak [11] to construct a Stein fillable contact structure $\xi_{Y}$ for each $Y \in \mathcal{F}$. The following is our main result.

Theorem 1.1. Let $Y \in \mathcal{F}$. Then, each Stein filling of $\left(Y, \xi_{Y}\right)$ has vanishing first Chern class and first Betti number. If $Y$ is elliptic then $\left(Y, \xi_{Y}\right)$ admits a unique Stein filling up to diffeomorphisms. If $Y$ is parabolic or hyperbolic then all the Stein fillings of $\left(Y, \xi_{Y}\right)$ share the same Betti numbers and fall into finitely many diffeomorphism classes.

As shown in Theorems 3.1 and 3.5, for some elliptic bundles and for parabolic and hyperbolic bundles in $\mathcal{F}$ the results of Theorem 1.1 hold more generally for minimal, strongly convex symplectic fillings rather than just for Stein fillings.

We are now going to describe the family $\mathcal{F}$. We will denote by $\mathbf{T}_{A}$ an oriented torus bundle over $S^{1}$ with monodromy specified by a matrix $A \in S L_{2}(\mathbb{Z})$. It is a well-known fact (cf. [19, Lemma 6.2]) that $\mathbf{T}_{A}$ is orientation-preserving diffeomorphic to $\mathbf{T}_{B}$ if and only if $A$ is conjugate in $S L_{2}(\mathbb{Z})$ to $B$. Moreover, $-\mathbf{T}_{A}$ is orientation-preserving diffeomorphic to $\mathbf{T}_{A^{-1}}$. A torus bundle $\mathbf{T}_{A}$ is

2010 Mathematics Subject Classification. 57R17, 57M50.

Key words and phrases. Stein fillings, torus bundles. 
called elliptic if $|\operatorname{tr} A|<2$, parabolic if $|\operatorname{tr}(A)|=2$ and hyperbolic if $|\operatorname{tr}(A)|>2$. Given $\left(d_{1}, \ldots, d_{m}\right) \in \mathbb{Z}^{m}, m \geq 1$, we define

$$
A\left(d_{1}, \ldots, d_{m}\right):=\left(\begin{array}{ll}
d_{m} & 1 \\
-1 & 0
\end{array}\right) \cdots\left(\begin{array}{cc}
d_{1} & 1 \\
-1 & 0
\end{array}\right) \in S L_{2}(\mathbb{Z}) .
$$

By [19, Proposition 6.3], if two $m$-tuples $d, d^{\prime} \in \mathbb{Z}^{m}$ as above are obtained from each other by a cyclic permutation then $\mathbf{T}_{A(d)}=\mathbf{T}_{A\left(d^{\prime}\right)}$ and, by [19. Theorem 6.1] $\mathbf{T}_{A}$ is hyperbolic with $\operatorname{tr}(A)<-2$ (respectively $\operatorname{tr}(A)>2$ ) if and only if $\mathbf{T}_{A}=\mathbf{T}_{-A(d)}$ (respectively $\mathbf{T}_{A}=\mathbf{T}_{A(d)}$ ) for some $d=\left(d_{1}, \ldots, d_{m}\right)$ with $d_{i} \geq 2$ for all $i$ and $d_{i} \geq 3$ for some $i$. Moreover, by the proof of [19, Theorem 6.1] and [19, Theorem 7.3], if

$$
d=(n_{1}+3, \underbrace{2, \ldots, 2}_{m_{1}}, n_{2}+3, \underbrace{2, \ldots, 2}_{m_{2}}, \ldots, n_{\ell}+3, \underbrace{2, \ldots, 2}_{m_{\ell}}), \quad m_{i}, n_{i} \geq 0,
$$

then $-\mathbf{T}_{ \pm A(d)}=\mathbf{T}_{ \pm A(\rho(d))}$, where

$$
\rho(d):=(m_{1}+3, \underbrace{2, \ldots, 2}_{n_{1}}, m_{2}+3, \underbrace{2, \ldots, 2}_{n_{2}}, \ldots, m_{\ell}+3, \underbrace{2, \ldots, 2}_{n_{\ell}}) .
$$

The following definition is inspired by a similar definition from [13]. A blowup of a sequence $\left(s_{1}, \ldots, s_{\ell}\right)$ of nonnegative integers is one of the following sequences:

$$
\left(s_{1}, \ldots, s_{i-1}, s_{i}+1,1, s_{i+1}+1, s_{i+2}, \ldots, s_{\ell}\right), \quad i=1, \ldots, \ell-1 .
$$

If a sequence $s^{\prime}$ is obtained from the sequence $s$ through a finite number of blowups, we also say that $s^{\prime}$ is a blowup of $s$. Given two sequences $s, c$ of length $\ell$, we write $s \prec c$ if $s_{i} \leq c_{i}$ for every $1 \leq i \leq \ell$, and we say that $d \in \mathbb{Z}^{m}$ is embeddable if $s \prec \rho(d)$ for some blowup $s$ of $(0,0)$.

We define $\mathcal{F}$ to be the set of torus bundles $Y$ over the circle such that one of following holds:

(1) $Y$ is elliptic;

(2) $Y$ is parabolic and $Y=-\mathbf{T}_{A(0,-n)}$ with $n \leq 4$;

(3) $Y$ is hyperbolic and $Y=-\mathbf{T}_{A(-c)}$ with $c \geq 3$;

(4) $Y$ is hyperbolic and $Y=\mathbf{T}_{-A(d)}$ with $d$ embeddable.

Combining Proposition 4.1 and Theorem 4.4 we obtain the following.

Theorem 1.2. Let $Y$ be a torus bundle of type $\mathbf{T}_{-A(\varepsilon)}$ with $\varepsilon \in\{-1,0,1\}$, $\mathbf{T}_{A(1)}$ or $\mathbf{T}_{-A(d)}$ with $d$ embeddable. Then, the contact structure $\xi_{Y}$ is the unique universally tight contact structure on $Y$ with vanishing Giroux torsion.

Theorem 1.2 has led us to formulate Conjecture 1 below. Before we can state it we need to introduce some notation. Let $(W, \omega)$ be a symplectic 4-manifold. A collection $D=C_{1} \cup \cdots \cup C_{n}$ of finitely many closed, embedded, symplectic surfaces in $W$ intersecting transversely and positively, and such that no three of them have a point in common will be called a symplectic divisor. When the symplectic form $\omega$ is part of a Kähler structure on $W$ and the surfaces $C_{i}$ are smooth, complex curves, we will call $D$ a complex divisor. When each $C_{i}$ is a 2 -sphere the divisor will be called spherical.

Conjecture 1. Let $(X, \omega)$ be a closed symplectic 4-manifold obtained as a symplectic blowup of $\mathbb{C P}^{2}$ with the standard Kähler form. Suppose that

$$
D=C_{1} \cup \cdots \cup C_{n} \subset X
$$

is a circular, spherical symplectic divisor such that $C_{i} \cdot C_{i} \in\{0,+1\}$ for some $i \in\{1, \ldots, n\}$. Then, any contact structure induced on the boundary of a concave neighbourhood of $D$ is universally tight. 
The paper is organized as follows. In Section 2] we use the work of Li-Mak [11] to prove Theorem 2.5. which says that, given a bundle $Y \in \mathcal{F}$, there exists a compact, symplectic 4-manifold with strictly $\omega$-concave boundary $\left(W_{Y}, \omega_{Y}\right)$ such that $\partial W_{Y}=-Y$ and $\left(W_{Y}, \omega_{Y}\right)$ embeds symplectically in a (deformation of) a blowup of the complex projective plane. The symplectic 4-manifolds $\left(W_{Y}, \omega_{Y}\right)$ are used in Section 3 to classify, up to diffeomorphisms, the Stein fillings of the contact 3-manifolds $\left(Y, \xi_{Y}\right)$, where $\xi_{Y}$ is the positive contact structure on $Y$ induced by the $\omega$-concave structure on the boundary of $W_{Y}$. Theorem 1.1 follows combining Theorems 3.1, 3.2 and 3.5. In Section 3 we also prove Proposition 3.6, showing the existence of infinitely many hyperbolic torus bundles $Y \in \mathcal{F}$ such that $\left(Y, \xi_{Y}\right)$ admits non-homotopy equivalent Stein fillings. In Section 4 we identify the contact structures $\xi_{Y}$ for some elliptic and hyperbolic bundles by proving Proposition 4.1 and Theorem 4.4, which imply Theorem 1.2. We also give explicit constructions of Stein fillings for $\left(Y, \xi_{Y}\right)$ when $Y$ is an elliptic torus bundle of type $\mathbf{T}_{-A(\varepsilon)}$ with $\varepsilon \in\{-1,0,1\}$, or $\mathbf{T}_{A(1)}$.

Acknowledgements. The authors wish to thank Youlin Li for suggesting that Theorem 3.5, originally proved for Stein fillings, might hold more generally for minimal, strongly convex symplectic fillings. The present work is part of the authors' activities within CAST, a Research Network Program of the European Science Foundation. Both authors were partially supported by the PRIN-MIUR research project 2010-11 "Varietà reali e complesse: geometria, topologia e analisi armonica", the first author was partially supported by the FIRB research project "Topologia e geometria di varietà in bassa dimensione" and by an ERC Exchange Grant.

\section{CONSTRUCTION OF SYMPLECTIC CAPS}

In this section we prove that for each torus bundle $Y$ belonging to the family $\mathcal{F}$ of Section 1 , there exists a compact, symplectic 4-manifold with strictly $\omega$-concave boundary $\left(W_{Y}, \omega_{Y}\right)$ such that $\partial W_{Y}=-Y$ and $\left(W_{Y}, \omega_{Y}\right)$ embeds symplectically in a (deformation of) a symplectic blowup of the standard symplectic $\mathbb{C P}^{2}$. We call a symplectic 4 -manifold $\left(W_{Y}, \omega_{Y}\right)$ as above a symplectic cap of $Y$. Our main tool to construct the symplectic 4-manifolds $W_{Y}$ will be the following theorem by $\mathrm{Li}$ and Mak.

Theorem 2.1 ([11, Theorem 1.3]). Let $D \subset\left(W, \omega_{0}\right)$ be a symplectic divisor. If the intersection form of $D$ is not negative definite and the restriction of $\omega_{0}$ to the boundary of a closed regular neighborhood of $D$ is exact, then $\omega_{0}$ can be deformed through a family of symplectic forms $\omega_{t}$ on $W$ keeping $D$ symplectic and such that, for any neighborhood $N$ of $D$, there is an $\omega_{1}$-concave neighborhood of $D$ inside $N$.

In the proof of Theorem 2.5 we will apply Theorem 2.1 to certain suitable spherical complex divisors in blowups of the complex plane $\mathbb{C P}^{2}$ endowed with their standard Kähler structure. We will obtain the divisors that we need by blowing up the following two basic configurations of immersed complex spheres in $\mathbb{C P}^{2}$ :

$(3 \ell)$ three complex lines in general position;

$\left(\ell \mathcal{C}_{2}\right)$ a line and a smooth conic in general position.

Regular neighborhoods of Configurations $(3 \ell)$ and $\left(\ell \mathcal{C}_{2}\right)$ are 4-dimensional plumbings given, in the notation of Neumann [19], by the graphs of Figure 1].

Elliptic bundles. Let $\mathbf{T}_{A}$ be a torus bundle with $|\operatorname{tr}(A)|<2$. It follows from the proof of [19. Proposition 2.1] (see [19, page 307]) that there are exactly six such torus bundles up to orientationpreserving diffeomorphisms, i.e. $\mathbf{T}_{ \pm A(\varepsilon)}$, with $\varepsilon=-1,0,1$ (here we are using Notation (1)). We claim that these bundles are the oriented boundaries of the six 4-dimensional plumbings given by Figure 2. Indeed, the proof of [19, Theorem 6.1] shows that the bundle given by the graph on the left 

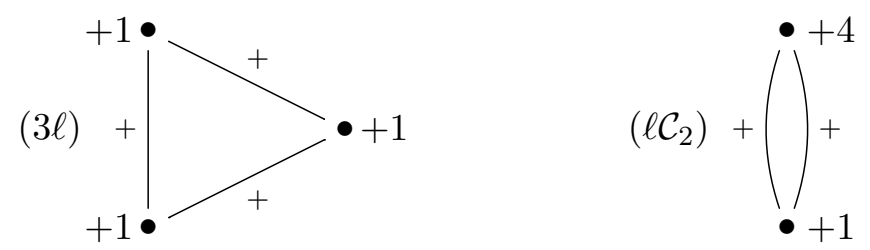

FIGURE 1. Plumbing graphs of the two basic configurations
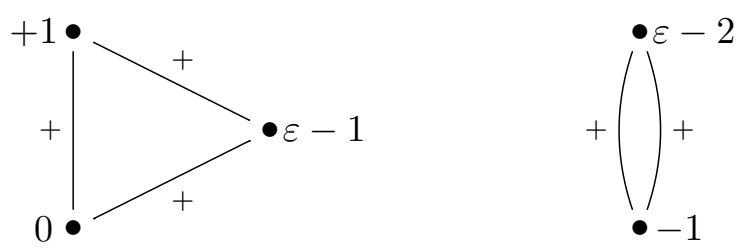

FIGURE 2. Plumbing graphs for elliptic torus bundles, $\varepsilon=-1,0,1$.

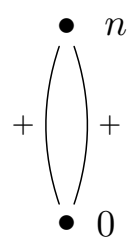

FiguRE 3. Plumbing graphs for parabolic bundles, $n \in \mathbb{Z}$.

of Figure 2 has monodromy

$$
A(1-\varepsilon, 0,-1)=\left(\begin{array}{cc}
\varepsilon & -1 \\
1 & 0
\end{array}\right)=-A(-\varepsilon),
$$

while the monodromy of the bundle given by the graph on the right is

$$
A(1,2-\varepsilon)=\left(\begin{array}{cc}
1-\varepsilon & -\varepsilon+2 \\
-1 & -1
\end{array}\right)=\left(\begin{array}{cc}
1 & -1 \\
0 & 1
\end{array}\right) A(-\varepsilon)\left(\begin{array}{cc}
1 & -1 \\
0 & 1
\end{array}\right)^{-1}
$$

Lemma 2.2. For $\varepsilon \in\{-1,0,1\}$ the graph on the left-hand side of Figure 2 is dual to the intersection graph of a spherical complex divisor $D \subset \mathbb{C P}^{2} \#(3-\varepsilon) \overline{\mathbb{C P}}^{2}$, while the graph on the right-hand side of Figure 2 is dual to the intersection graph of a spherical complex divisor $D \subset \mathbb{C P}^{2} \#(8-\varepsilon) \overline{\mathbb{C P}}^{2}$.

Proof. (1) Let the $\ell_{1}, \ell_{2}, \ell_{3}$ be the three generic lines of the basic configuration $(3 \ell)$ inside $\mathbb{C P}^{2}$ with the Fubini-Study form. Blow up $\mathbb{C P}^{2}$ at one generic point of $\ell_{2}$ and at $2-\varepsilon$ generic points of $\ell_{3}$, and let $D \subset \mathbb{C P}^{2} \#(3-\varepsilon) \overline{\mathbb{C P}}^{2}$ be the proper transform of $\ell_{1} \cup \ell_{2} \cup \ell_{3}$.

(2) $D$ is obtained as the proper transform of the configuration $\ell \mathcal{C}_{2}$ in $\mathbb{C P}^{2}$ blown up at two generic points of the line and at $6-\varepsilon$ generic points of the conic.

Parabolic bundles. Arguing as in the proof of [19, Theorem 6.1] and using Notation (1) it is easy to check that the boundary of the plumbing given by the graph of Figure 3 is a (parabolic) torus bundle with monodromy $A(0,-n)=-\left(\begin{array}{ll}1 & n \\ 0 & 1\end{array}\right)$.

Lemma 2.3. For every integer $n \leq 4$ the graph of Figure 3 is dual to the intersection graph of a spherical complex divisor $D \subset \mathbb{C P}^{2} \#(5-n) \overline{\mathbb{C P}}^{2}$. 
Proof. When $n \leq 4$ the graph of Figure 3 is the intersection graph of the proper transform of the basic configuration $\left(\ell \mathcal{C}_{2}\right)$ in $\mathbb{C P}^{2}$, obtained by blowing up at $4-n$ generic points of the conic $\mathcal{C}$ and one generic point of the line $\ell$.

Hyperbolic bundles. Let $\mathbf{T}_{A}$ by a hyperbolic bundle with $\operatorname{tr}(A)<-2$. As explained in Section 1 , $\mathbf{T}_{A}=\mathbf{T}_{-A(d)}$, where $d=\left(d_{1}, \ldots, d_{m}\right) \in \mathbb{Z}^{m}, d_{i} \geq 2$ for all $i$ and $d_{i} \geq 3$ for some $i$, and $-\mathbf{T}_{-A(d)}=\mathbf{T}_{-A(\rho(d))}$, where $\rho(d)=\left(c_{1}, \ldots, c_{\ell}\right)$ is defined by Equation (2). Moreover, by [19. Theorem 7.1] $\mathbf{T}_{-A(\rho(d))}$ is the boundary of the 4-dimensional plumbing given by Figure 4 . Using

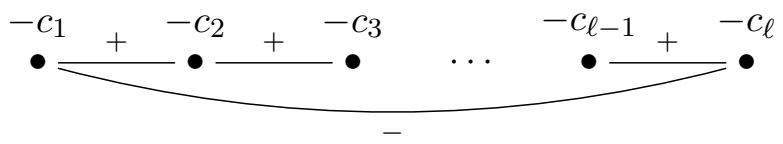

FiguRE 4. Plumbing graphs for $-\mathbf{T}_{A}$ with $\mathbf{T}_{A}$ hyperbolic and $\operatorname{tr}(A) \leq-3$.

Neumann's plumbing calculus (i.e. [19, Proposition 2.1]) it is easy to check that when $\ell>1$ the bundle $-\mathbf{T}_{A}=\mathbf{T}_{-A(\rho(d))}$ is also the oriented boundary of the plumbing given by the graph on the left of Figure 5, while when $\ell=1$ it is given by the graph on the right of the same figure (observe that in this case $c_{1} \geq 3$ by (2)).

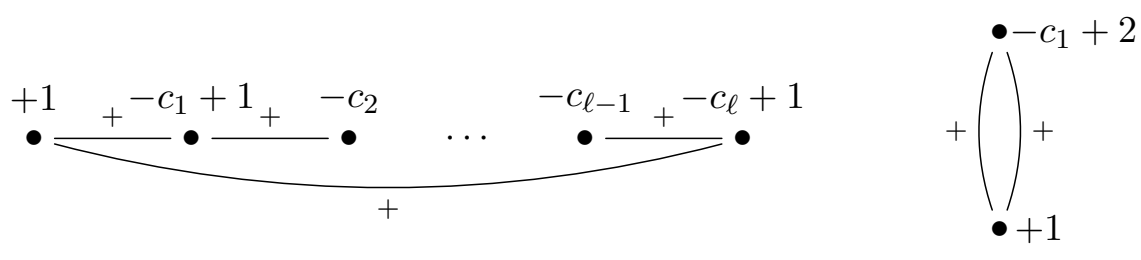

FIGURE 5. Alternative plumbing graphs for $-\mathbf{T}_{A}$ with $\operatorname{tr}(A) \leq-3$.

Lemma 2.4. Let $d=\left(d_{1}, \ldots, d_{m}\right) \in \mathbb{Z}^{m}$ with $d_{i} \geq 2$ for all $i, d_{i} \geq 3$ for some $i$ and let $\left(c_{1}, \ldots, c_{\ell}\right)=\rho(d)$. Suppose that either $d$ is embeddable or $\ell=1$. Then, there is a spherical complex divisor in a blowup of $\mathbb{C P}^{2}$ whose dual intersection graph equals the graph on the left of Figure 5 if $d$ is embeddable and the graph on the right of the same figure if $\ell=1$.

Proof. When $\ell=1$ the graph on the right of Figure 5 is dual to a spherical complex divisor $D \subset \mathbb{C P}^{2} \#\left(c_{1}+2\right) \overline{\mathbb{C P}}^{2}$ consisting of the proper transforms of the line and the conic of the basic configuration $\left(\ell \mathcal{C}_{2}\right)$. This is easily shown as in the proof of Lemma 2.3 .

When $d$ is embeddable the graph on the left of Figure 5 is dual to a spherical complex divisor inside a blowup of $\mathbb{C P}^{2}$ consisting of the proper transforms of the three lines of the basic configuration $(3 \ell)$. In order to see this, consider the configuration $D_{0}=(3 \ell)$ of three lines in general position in $\mathbb{C P}^{2}$, and let $\ell$ be one of the lines. The line $\ell$ will correspond to the sphere with self-intersection +1 in the final divisor. Associate the string $s^{0}=(0,0)$ to $D_{0}$ and define inductively configurations $D_{k}$, $k \geq 0$, as follows. By assumption there is a sequence of blowups $s^{0} \rightsquigarrow s^{1} \rightsquigarrow \cdots \rightsquigarrow s^{n}=s$. For each $k=0, \ldots, n-1$ the blowup $s^{k} \rightsquigarrow s^{k+1}$ determines in a natural way a symplectic blowup at a nodal point of $D_{k}$ not lying on $\ell$. We define $D_{k+1}$ as the total transform of $D_{k}$. The self-intersection of the $i$-th sphere in $D_{k}$ is $-s_{i}^{k}$, except for $i=1$ and $i=k+1$, in which case the self intersection is $-s_{i}^{k}+1$. Finally, for each $i$ we blow up $c_{i}-s_{i}$ times at generic points of the $i$-th component of $D_{n}$ and take the resulting proper transform. 
Existence of the symplectic caps. We are now ready to apply Theorem 2.1 in order to establish the following theorem, which is the main result of this section.

Theorem 2.5. Let $Y$ be a torus bundle over $S^{1}$. Then, $Y$ admits a symplectic cap $W_{Y}$ which is a closed regular neighborhood of a spherical complex divisor $D$ in a deformation of a blowup of $\mathbb{C P}^{2}$ with its standard Kähler form, if one of the following conditions is verified:

(1) $Y$ is elliptic and $Y=\mathbf{T}_{A(\varepsilon)}$, with $\varepsilon \in\{-1,0,1\}$; in this case D has intersection graph dual to the graph on the left of Figure 2 .

(2) $Y$ is elliptic and $Y=\mathbf{T}_{-A(\varepsilon)}$, with $\varepsilon \in\{-1,0,1\}$; in this case $D$ has intersection graph dual to the graph on the right of Figure 2.

(3) $Y$ is parabolic and $Y=-\mathbf{T}_{A(0,-n)}$ with $n \leq 4$; in this case $D$ has intersection graph dual to the graph of Figure 3 .

(4) $Y$ is hyperbolic and $Y=-\mathbf{T}_{A(-c)}$ with $c \geq 3$; in this case $D$ has intersection graph dual the graph on the right of Figure 5 .

(5) $Y$ is hyperbolic $Y=\mathbf{T}_{-A(d)}$ with d embeddable; in this case D has intersection graph dual to the graph on the left of Figure 5

Moreover, for each of the bundles $Y$ specified above we have $b_{1}(Y)=1$, and the contact 3-manifold $\left(Y, \xi_{Y}\right)$ admits a Stein filling diffeomorphic to the complement of a regular neighborhood of the corresponding spherical symplectic divisor D constructed in one of Lemmas 2.22 .3 or 2.4.

Proof. We would like to apply Theorem 2.1 to the complex divisors $D$ appearing in Lemmas 2.2,2.3 and 2.4. Recall that $D$ is contained in a blowup $X$ of the standard Kähler $\mathbb{C P}^{2}$. It is easy to check using e.g. the statement of [19. Proposition 2.1] that $-\mathbf{T}_{A(\varepsilon)}=\mathbf{T}_{-A(-\varepsilon)}$ for each $\varepsilon \in\{-1,0,+1\}$. Thus, in view of the three lemmas and the discussions preceding them, to apply Theorem 2.1 it suffices to show that (i) the restriction of the Kähler form $\omega_{0}$ to the boundary of a closed regular neighborhood of $D$ is exact and (ii) for each graph $\Gamma$ mentioned in the statement the corresponding intersection matrix $Q_{\Gamma}$ is not negative definite. Viewing $\mathbf{T}_{A}$ as the union of two copies of a 2-torus times an interval and applying Mayer-Vietoris yields the exact sequence

$$
\cdots \longrightarrow \mathbb{Z}^{2} \oplus \mathbb{Z}^{2} \stackrel{\left(\begin{array}{ll}
I & I \\
A & I
\end{array}\right)}{\longrightarrow} \mathbb{Z}^{2} \oplus \mathbb{Z}^{2} \longrightarrow H_{1}\left(\mathbf{T}_{A} ; \mathbb{Z}\right) \longrightarrow \mathbb{Z} \longrightarrow 0
$$

This immediately implies:

$$
H_{1}\left(\mathbf{T}_{A} ; \mathbb{Z}\right) \cong \mathbb{Z} \oplus \operatorname{coker}(A-I) \text {. }
$$

Since $A \in S L_{2}(\mathbb{Z}), A-I$ can be singular only if $A$ is parabolic with $\operatorname{tr}(A)=2$. But we are considering only parabolic bundles of the form $\mathbf{T}_{A(0,-n)}$, and $\operatorname{tr}(A(0,-n))=\operatorname{tr}\left(-\left(\begin{array}{ll}1 & n \\ 0 & 1\end{array}\right)\right)=-2$, therefore in all our cases coker $(A-I)$ is a torsion group. This shows that $b_{1}(Y)=1$ for each torus bundle given in the statement. Let $W$ be a closed regular neighborhood of the divisor corresponding to $\mathbf{T}_{A}$ and given by one of Lemmas 2.2, 2.3 and 2.4. By construction we have $\partial W=-\mathbf{T}_{A}$, and the homology exact sequence of the pair $\left(W, \mathbf{T}_{A}\right)$ contains the exact sequence

$$
\cdots \longrightarrow H_{2}\left(\mathbf{T}_{A} ; \mathbb{Z}\right) \longrightarrow H_{2}(W ; \mathbb{Z}) \stackrel{Q_{\Gamma}}{\longrightarrow} H_{2}\left(W, \mathbf{T}_{A} ; \mathbb{Z}\right) \longrightarrow H_{1}\left(\mathbf{T}_{A} ; \mathbb{Z}\right) \longrightarrow H_{1}(W ; \mathbb{Z}) \longrightarrow 0 .
$$

From this sequence we deduce

$$
H_{1}\left(\mathbf{T}_{A} ; \mathbb{Z}\right) \cong \mathbb{Z} \oplus \operatorname{coker}\left(Q_{\Gamma}\right),
$$

and therefore $\operatorname{coker}\left(Q_{\Gamma}\right) \cong \operatorname{coker}(A-I)$. Since coker $(A-I)$ is a torsion group we conclude that $Q_{\Gamma}$ is nonsingular, hence the map $H_{2}\left(\mathbf{T}_{A} ; \mathbb{Z}\right) \rightarrow H_{2}(W ; \mathbb{Z})$ vanishes. This implies that if $[F] \in$ $H_{2}\left(\mathbf{T}_{A} ; \mathbb{R}\right)$ is the class carried by a torus fiber of the fibration $\mathbf{T}_{A} \rightarrow S^{1}$ and $i_{*}: H_{2}\left(\mathbf{T}_{A} ; \mathbb{R}\right) \rightarrow$ 
$H_{2}(X ; \mathbb{R})$ is the map induced by inclusion, we have $i_{*}([F])=0$. Therefore, when we evaluate on $[F]$ the restriction of $\left[\omega_{0}\right] \in H^{2}(X ; \mathbb{R})$ to $H^{2}\left(\mathbf{T}_{A} ; \mathbb{R}\right)$ we get

$$
\left\langle i^{*}\left[\omega_{0}\right],[F]\right\rangle=\left\langle\left[\omega_{0}\right], i_{*}[F]\right\rangle=0 .
$$

Since $H_{2}\left(\mathbf{T}_{A} ; \mathbb{R}\right)$ is generated by $[F]$ we conclude $i^{*}\left(\left[\omega_{0}\right]\right)=0$, i.e. the restriction of $\omega_{0}$ to $\mathbf{T}_{A}$ is exact. Finally, $Q_{\Gamma}$ is never negative definite, as one can easily check by looking at the corresponding intersection graph $\Gamma$. We can therefore apply Theorem 2.1 as explained at the beginning. Theorem 2.1 implies that there is a one-parameter family of symplectic forms on $X$ which interpolates between the Kähler form $\omega_{0}$ and a symplectic form $\omega_{1}$, with the property that any neighborhood of $D$ contains an $\omega_{1}$-concave neighborhood. Since $X$ is compact, a Moser-type argument produces a diffeomorphism $\phi: X \rightarrow X$ such that $\phi^{*} \omega_{1}=\omega_{0}$. Pushing forward via $\phi$ the integrable complex structure $J_{0}$ compatible with $\omega_{0}$ yields an integrable complex structure $J_{1}$ compatible with $\omega_{1}$. Setting $Y=\mathbf{T}_{A}$, we obtain a symplectic cap $W_{Y}$ from any $\omega_{1}$-concave neighborhood of $D$. Moreover, the complement $X^{\prime}$ in $X$ of the interior of $W_{Y}$, endowed with the complex structure $J_{1}$, is a strictly pseudo-convex surface in the sense of [2]. By [2, Theorem 2'] there is a small deformation of $X^{\prime}$ which is a Stein filling of $\left(Y, \xi_{Y}\right)$. This concludes the proof.

\section{Fillings}

In this section we prove Theorem 1.1. The theorem will follow combining Theorems 3.1, 3.2 and 3.5 below. At the end of the section we prove Proposition 3.6, which shows that the family $\left\{\left(Y, \xi_{Y}\right) \mid Y \in \mathcal{F}\right\}$ contains infinitely many contact hyperbolic torus bundles admitting non-homotopy equivalent Stein fillings with even intersection forms and the same Betti numbers.

Theorem 3.1. Let $Y$ be a torus bundle over $S^{1}$ such that one of the following holds:

(1) $Y$ is elliptic and $Y=\mathbf{T}_{A(\varepsilon)}$ with $\varepsilon \in\{-1,0,+1\}$;

(2) $Y$ is hyperbolic and $Y=-\mathbf{T}_{A(-c)}$ with $c \geq 3$;

(3) $Y$ is hyperbolic and $Y=\mathbf{T}_{-A(d)}$ with dembeddable.

Then,

- each minimal, strongly convex symplectic filling of $\left(Y, \xi_{Y}\right)$ has vanishing first Chern class and first and third Betti numbers;

- in Cases 1 and 2 the contact 3-manifold $\left(Y, \xi_{Y}\right)$ admits a unique minimal, strongly convex symplectic filling up to diffeomorphisms;

- in Case 3 all the minimal, strongly convex symplectic fillings of $\left(Y, \xi_{Y}\right)$ share the same second Betti number and fall into finitely many diffeomorphism classes.

Proof. Cases 1, 2 and 3 of the statement correspond respectively to Cases 1,4 and 5 of Theorem 2.5 . In all cases the dual intersection graph of the symplectic divisor $D \subset W_{Y}$ contains at least three vertices, one of which has weight +1 . This latter vertex corresponds to an embedded symplectic sphere $S \subset W_{Y}$ with self-intersection +1 .

We first deal with Cases 1 and 3. If we blow up symplectically $W_{Y}$ at a nodal point of $D$ away from $S$, the total transform $\widetilde{D} \subset \widehat{W}_{Y}:=W_{Y} \# \overline{\mathbb{C P}}^{2}$ contains at least four spheres. Notice that the boundary of the symplectic cap $\widehat{W}_{Y}$ is still strongly $\omega$-concave and the contact structure induced on the boundary is still $\xi_{Y}$. Let $P$ be a minimal, strongly convex symplectic filling of $\left(Y, \xi_{Y}\right)$. Since the boundary of $\widehat{W}_{Y}$ is strongly $\omega$-concave, we can construct a closed symplectic 4-manifold $(X, \omega)$ by symplectically gluing the cap $\widehat{W}_{Y}$ and $P$ together along $Y$ after possibly rescaling the symplectic form on one of the two pieces. Since $\widetilde{D} \subset \widehat{W}_{Y}, X$ contains an embedded symplectic +1 -sphere $S$. Hence, by [16, Theorem 1.1 and Corollary 1.6] $X$ is symplectomorphic to 
a symplectic blowup of $\mathbb{C P}^{2}$ endowed with the standard Kähler form, in such a way that $S$ represents the hyperplane class. Since $b_{1}(Y)=b_{1}\left(\widehat{W}_{Y}\right)=1$ and $b_{1}(X)=b_{3}\left(\widehat{W}_{Y}\right)=0$, the Mayer-Vietoris exact sequence of homology groups associated to the decomposition $X=\widehat{W}_{Y} \cup P$ shows that $b_{1}(P)=b_{3}(P)=0$. We can choose an $\omega$-tame almost complex structure $J$ on $X$ which makes all the symplectic spheres in $\widetilde{D}$ pseudo-holomorphic. Let $S^{\prime} \subset \widetilde{D}$ be one of the two symplectic spheres intersecting $S$. By construction $\widetilde{D}^{\prime}:=\widetilde{D} \backslash S^{\prime}$ consists of a chain of $k$ symplectic spheres for some $k \geq 3$; their self-intersection numbers are $\left(1,1-b_{1},-b_{2}, \ldots,-b_{k}\right)$. By [12, Theorem 4.2] there is a sequence of symplectic blowdowns of $X$ to $\mathbb{C P}^{2}$ such that $\widetilde{D}^{\prime}$ blows down to the union of two lines $\ell \cup \ell^{\prime} \subset \mathbb{C P}^{2}$. Moreover, at each step the almost complex structure descends, and, since $P$ is minimal, the exceptional divisor that we blow down either intersects the configuration positively once or belongs to the configuration. During this process the sphere $S^{\prime}$ blows down to a smoothly embedded symplectic sphere intersecting both $\ell$ and $\ell^{\prime}$ exactly once, hence $S^{\prime}$ blows down to a line. It follows that $\widetilde{D}$ blows down to a generic configuration $C$ of three generically embedded symplectic spheres which are pseudo-holomorphic with respect to an almost complex structure tamed by the standard Kähler form on $\mathbb{C P}^{2}$. By a theorem of Gromov [9] (see also [23, Lemma 2.7]) the embedding of such three symplectic spheres in general position is unique up to isotopy. Therefore, up to isotopy we may assume that $C$ coincides with a basic configuration $(3 \ell)$ of three complex lines. This means that the configuration $\widetilde{D}$ is obtained from $(3 \ell)$ via a sequence of blowups. Since the homology class carried by the divisor $(3 \ell)$ is Poincaré dual to $c_{1}\left(\mathbb{C P}^{2}\right)$, we conclude that $c_{1}(X)$ is Poincaré dual to $[\widetilde{D}]$. In particular, $c_{1}(P)=0$, the total number of blowups must be $N=9-[\widetilde{D}]^{2}$, and the second Betti number of $P$ is determined to be $b_{2}(P)=N+1-b_{2}\left(W_{Y}\right)$. The homology classes carried by the symplectic spheres comprising $\widetilde{D}$ are determined as in [12, Theorem 4.2], up to a little proviso: one needs to pay attention to the way $S^{\prime}$ intersects the other sphere $S^{\prime \prime}$ which intersects $S$ nontrivially. Since we made sure that $\widetilde{D}$ contains at least four spheres, $S^{\prime}$ and $S^{\prime \prime}$ intersect trivially. This implies that if we denote by $h$ the hyperplane class and by $e_{i}$ the classes of the exceptional divisors, since both $\left[S^{\prime}\right]$ and $\left[S^{\prime \prime}\right]$ are of the form $h+\sum c_{i} e_{i}$ and there exists exactly one index $i$ such that the coefficient $c_{i}$ in both expressions is nonvanishing (and equal to -1 ). Indeed, one can check that the exceptional divisor corresponding to $e_{i}$ comes from blowing up two lines of $(3 \ell)$ at their intersection point, and that there is a divisor in $\widetilde{D}$ carrying a class $e_{i}-\sum_{j \neq i} x_{j} e_{j}$ for some $x_{j} \geq 0$. But there are clearly finitely many possible sequences of blowups compatible with the above construction, and exactly one (up to reordering) in Case 1. It follows that the diffeomorphism type of the complement of a neighborhood of $\widetilde{D} \hookrightarrow X \cong \mathbb{C P}^{2} \# N \overline{\mathbb{C P}}^{2}$ is uniquely determined in Case 1 , and determined up to finitely many possiblities in Case 3 . This concludes the proof in Cases 1 and 3 .

The proof in Case 2 is quite similar, so we just outline the differences with the previous cases. In this case we do not blow up $W_{Y}$ at the beginning, so we consider directly the closed symplectic 4-manifold $X=W_{Y} \cup P$, where $P$ is a minimal, strongly convex symplectic filling. By the same argument as above, $X$ is symplectomorphic to a blowup of $\mathbb{C P}^{2}$ and $b_{1}(P)=b_{3}(P)=0$. The symplectic divisor $D$ is a union of smoothly embedded symplectic spheres $S$ and $S^{\prime}$, where $S \cdot S=+1, S^{\prime} \cdot S^{\prime}=-c_{1}+2$ with $c_{1} \geq 3$ and $[S]=h$, where $h$ is the hyperplane class of $X$. Moreover, the adjunction formula for $S^{\prime}$ and the fact that $S \cdot S^{\prime}=2$ imply $\left[S^{\prime}\right]=2 h-\sum_{i} e_{i}$, where the classes $e_{i}$ are the exceptional classes. As before, this implies that $D$ blows down to a configuration of two symplectic spheres in $\mathbb{C P}^{2}$, one representing $h$ and the other $2 h$. But the moduli space of smoothly embedded symplectic curves in the class $2 h$ in $\mathbb{C P}^{2}$ is connected and each pair of points determines a unique pseudo-holomorphic line [9], hence up to isotopy we may assume that $D$ blows down to a basic configuration $\left(\ell \mathcal{C}_{2}\right)$. Since there is clearly a unique way (up to reordering) to blow up $\left(\ell \mathcal{C}_{2}\right)$ to get $D$, the diffeomorphism type of $P$ is uniquely determined. Since the homology class 
carried by $\left(\ell \mathcal{C}_{2}\right)$ is Poincaré dual to $c_{1}\left(\mathbb{C P}^{2}\right)$, we conclude as in Cases 1 and 3 that $c_{1}(X)$ is Poincaré dual to $[D]$ and $c_{1}(P)=0$.

Theorem 3.2. Let $Y$ be an elliptic torus bundle over $S^{1}$ of the form $Y=\mathbf{T}_{-A(\varepsilon)}$, with $\varepsilon \in\{-1,0,1\}$. Then, all Stein fillings of $\left(Y, \xi_{Y}\right)$ have vanishing first Chern class and first Betti number, and they are pairwise orientation-preserving diffeomorphic.

Proof. Let $(P, J)$ be a Stein filling of $\left(Y, \xi_{Y}\right)$. We start by arguing that $c_{1}(P)=0$. By Honda's classification [10], there is only one isotopy class of contact structures without Giroux torsion on an elliptic bundle $Y$. Since fillable contact structures have no Giroux torsion [5], both $\xi_{Y}$ and its conjugate $\overline{\xi_{Y}}$ belong to this isotopy class. Since $\bar{J}$ is another Stein structure on $P$ which fills $\overline{\xi_{Y}}$, applying [14, Theorem 1.2] we conclude $c_{1}(P)=0$.

The elliptic bundles $Y$ of type $\mathbf{T}_{-A(\varepsilon)}$ are considered in Case 2 of Theorem 2.5, which says that $Y$ has a symplectic cap $W_{Y}$ and the corresponding divisor $D$ has intersection graph $\Gamma$ dual to the graph on the right of Figure 2 . Therefore, there are smoothly embedded symplectic spheres $S_{1}, S_{2} \subset W_{Y}$ with $S_{1} \cdot S_{1}=-1, S_{2} \cdot S_{2}=\varepsilon-2$ and $S_{1} \cdot S_{2}=+2$. The exceptional symplectic sphere $S_{1} \subset W_{Y}$ allows us to write $W_{Y}=W_{Y}^{\prime} \# \overline{\mathbb{C P}}^{2}$, where $W_{Y}^{\prime}$ is a symplectic cap of $Y$ diffeomorphic to a closed neighborhood of an immersed nodal symplectic sphere $S_{2}^{\prime}$ with self-intersection $2+\varepsilon$. Moreover, it is easy to check that $c_{1}\left(W_{Y}^{\prime}\right)=P D\left(S_{2}^{\prime}\right)$.

Let $X^{\prime}$ be a closed symplectic 4 -manifold obtained by gluing the symplectic cap $W_{Y}^{\prime}$ to $P$ along their common boundary. First of all we want to argue that $b_{2}^{+}\left(X^{\prime}\right)=1$. Smoothing the singularity of $S_{2}^{\prime}$ we obtain a smoothly embedded 2-torus with self-intersection $2+\varepsilon>0$ inside $X^{\prime}$. But such a torus violates the adjunction inequality, which is known to hold for closed, symplectic 4-manifolds with $b_{2}^{+}>1$. Therefore we must have $b_{2}^{+}\left(X^{\prime}\right)=1$.

Now we claim that $c_{1}\left(X^{\prime}\right)=P D\left(S_{2}^{\prime}\right) \in H^{2}\left(X^{\prime}\right)$ (we are going to use $\mathbb{Z}$ coefficients throughout the proof). Observe that each of the cohomology classes $c_{1}\left(X^{\prime}\right)$ and $P D\left(S_{2}^{\prime}\right)$ both restrict as 0 to $H^{2}(P)$ and as $c_{1}\left(W_{A}^{\prime}\right)$ to $H^{2}\left(W_{Y}^{\prime}\right)$. Therefore, in order to show that they are equal it suffices to check that the map $H^{2}\left(X^{\prime}\right) \rightarrow H^{2}(P) \oplus H^{2}\left(W_{Y}^{\prime}\right)$ appearing in the Mayer-Vietoris sequence for the decomposition $X^{\prime}=P \cup W_{Y}^{\prime}$ is injective. This follows from the fact that the restriction map $H^{1}\left(W_{Y}^{\prime}\right) \rightarrow H^{1}(Y)$ is surjective. The latter is equivalent, by Poincaré duality and the homology exact sequence of the pair $\left(W_{Y}^{\prime}, Y\right)$, to the fact that the map $H_{2}(Y) \rightarrow H_{2}\left(W_{Y}^{\prime}\right)$ induced by inclusion is the zero map, which follows immediately from the fact that $S_{2}^{\prime} \cdot S_{2}^{\prime} \neq 0$. Therefore the claim is established.

Observe that, if $\omega$ is the symplectic form on $X^{\prime}$, the claim implies

$$
c_{1}\left(X^{\prime}\right) \cdot[\omega]=\int_{S_{2}^{\prime}} \omega>0 .
$$

Thus, we can apply Theorem [15, Theorem B], which says that if $(X, \omega)$ is a closed, symplectic 4-manifold with $b_{2}^{+}(X)=1$ and $K_{X} \cdot[\omega]<0$ then $X$ is either rational (i.e. a blowup of $\mathbb{C P}^{2}$ ) or ruled, i.e. a symplectic sphere bundle. We conclude that $X^{\prime}$ is either rational or ruled, and we claim that $X^{\prime}$ cannot be ruled. In fact, suppose the contrary, and let $B$ be the base. Observe that $\chi\left(X^{\prime}\right)=\chi(B) \chi\left(S^{2}\right)=2 \chi(B)$. Moreover, from the Mayer-Vietoris sequence of the decomposition $X^{\prime}=N \cup\left(X^{\prime} \backslash N\right)$, where $N$ is a regular neighborhood of a fiber, it is easy to deduce that $1 \leq b_{2}\left(X^{\prime}\right) \leq 2$. Since the class of a symplectic fiber is nontrivial and of square zero, this immediately implies $\sigma\left(X^{\prime}\right)=0$. Therefore we have $c_{1}\left(X^{\prime}\right)^{2}=3 \sigma\left(X^{\prime}\right)+2 \chi\left(X^{\prime}\right)=4 \chi(B)$, contradicting the fact that $c_{1}\left(X^{\prime}\right)^{2}=2+\varepsilon$ with $\varepsilon \in\{-1,0,1\}$. We conclude that $X^{\prime}$ must be rational, i.e. symplectomorphic to an $r$-fold blowup of $\mathbb{C P}^{2}$. This implies $c_{1}^{2}\left(X^{\prime}\right)=9-r$, with $r \in\{6,7,8\}$, and therefore $c_{1}\left(X^{\prime}\right)=P D\left(S_{2}^{\prime}\right)=3 h-e_{1}-\cdots-e_{r}$, where $h$ is the hyperplane class 
and the classes $e_{i}$ the exceptional classes. Arguing as in the proof of Theorem 3.1 we can deduce that $S_{2}^{\prime}$ is the proper transform of an $r$-fold blowup of a nodal pseudo-holomorphic cubic in $\mathbb{C P}^{2}$. Since the moduli space of pseudo-holomorphic nodal cubics is connected [4, Theorem 13], it follows that the diffeomorphism type of $P$ is determined, and given by the complement of a neighborhood of the strict transform of a nodal holomorphic cubic in an $r$-fold blowup of $\mathbb{C P}^{2}$, with $r \in\{6,7,8\}$. Finally, using the fact that $b_{1}\left(W_{Y}^{\prime}\right)=1$ and $b_{1}\left(X^{\prime}\right)=0$ and arguing as in the proof of Theorem 3.1 shows that $b_{1}(P)=0$.

Lemma 3.3. Let $(X, \omega)$ be a closed, symplectic 4-manifold containing the configuration $\Sigma$ of two transverse symplectic spheres described by the plumbing of Figure 3 for $n=4$. If $X \backslash \Sigma$ is minimal, then either $X=\mathbb{C P}^{2} \# \overline{\mathbb{C P}}^{2}$ and $\Sigma$ is the strict transform of the configuration $\left(\ell C_{2}\right)$ blown up at a generic point of the line, or $X=S^{2} \times S^{2}$ and $\Sigma$ is the union of $S^{2} \times\{*\}$ and the graph of a holomorphic map $S^{2} \rightarrow S^{2}$ of degree 2. In both cases the first Chern class of X vanishes on $X \backslash \Sigma$.

Proof. Let $S_{1}, S_{2}$ be the two symplectic spheres of $\Sigma$, with $S_{1} \cdot S_{1}=0, S_{1} \cdot S_{2}=+2$ and $S_{2} \cdot S_{2}=+4$. By [16, Corollary 1.5], the pair $\left(X, S_{2}\right)$ is an $r$-fold blowup of either $\left(\mathbb{C P}^{2}, q\right)$ or $\left(S^{2} \times S^{2}, \Gamma\right)$, where $q$ is a conic, $\Gamma$ is the graph of a holomorphic map $S^{2} \rightarrow S^{2}$ of degree 2, and the exceptional spheres in $X$ are all disjoint from $S_{2}$. Call $e_{1}, \ldots, e_{r}$ the exceptional homology classes.

If $\left(X, S_{2}\right)$ is a blowup of $\left(\mathbb{C P}^{2}, q\right)$, let $h$ be the homology class of a complex line in $\mathbb{C P}^{2}$, so that $\left[S_{2}\right]=2 h$. The conditions $S_{1} \cdot S_{2}=2$ and $S_{1} \cdot S_{1}=0$ imply $\left[S_{1}\right]=h-\sum x_{i} e_{i}$, with exactly one index $i$ such that $x_{i}=1$, while $x_{j}=0$ for each $j \neq i$. By positivity of intersections [17] each exceptional sphere is disjoint from $\Sigma$, and since $X \backslash \Sigma$ is minimal this means that $r=i=1$. Moreover, the Poincaré dual of $c_{1}(X)$ equals $\left[S_{1}\right]+\left[S_{2}\right]$, and the statement is proved in this case.

If $\left(X, S_{2}\right)$ is a blowup of $\left(S^{2} \times S^{2}, \Gamma\right)$ then $\left[S_{2}\right]=2 s+f$, where $s=\left[S^{2} \times\{*\}\right]$ and $f=\left[\{*\} \times S^{2}\right]$. We have $\left[S_{1}\right]=a s+b f-\sum x_{i} e_{i}$, with $a, b, x_{i} \geq 0$ by positivity of intersections. Imposing that $S_{1} \cdot S_{2}=2$ we obtain $a+2 b=2$, therefore either $(a, b)=(2,0)$ or $(a, b)=(0,1)$. Imposing that $S_{1} \cdot S_{1}=0$ we obtain that $x_{i}=0$ for each $i$. Finally, the adjunction formula excludes the case $(a, b)=(2,0)$, hence $\left[S_{1}\right]=f$, and positivity of intersections implies that each exceptional sphere in $X$ is disjoint from $\Sigma$. Therefore, since $X \backslash \Sigma$ is minimal, in this case we have $r=0$. As in the previous case $c_{1}(X)=\left[S_{1}\right]+\left[S_{2}\right]$, and the statement is proved.

Lemma 3.4. For $n>4$ the configuration of two symplectic spheres described by the plumbing of Figure 3 does not embed in any closed, symplectic 4-manifold.

Proof. Suppose by contradiction that there exists a closed symplectic 4-manifold $\left(X^{0}, \omega^{0}\right)$ containing two embedded spheres $S_{1}^{0}$, $S_{2}^{0}$ of self-intersection 0 and $n$ respectively, with $S_{1}^{0} \cdot S_{2}^{0}=2$. Let $(X, \omega)$ be the symplectic 4-manifold obtained by blowing up $X^{0}$ at $n-4$ generic points of $S_{2}^{0}$. Let $e_{1}, \ldots, e_{n-4}$ be the corresponding exceptional classes, and $S_{1}, S_{2}$ the proper transforms of $S_{1}^{0}$ and $S_{2}^{0}$, respectively. Now $S_{1} \cdot S_{1}=0, S_{2} \cdot S_{2}=4$ and $S_{1} \cdot S_{2}=2$. Notice that $\left[S_{2}\right] \cdot e_{1}=1$, and therefore the homology class $\left[S_{2}\right]$ cannot be even. By Lemma 3.3. and since $\left[S_{2}\right]$ is not even, $(X, \omega)$ must be a blowup of $S^{2} \times S^{2}$ with $\left[S_{1}\right]=f,\left[S_{2}\right]=2 s+f$, where $s=\left[S^{2} \times\{*\}\right]$ and $f=\left[\{*\} \times S^{2}\right]$ and all the exceptional spheres are disjoint from $S_{1} \cup S_{2}$. This contradicts the fact that $\left[S_{2}\right] \cdot e_{1}=1$.

Theorem 3.5. Let $Y$ be a parabolic torus bundle over $S^{1}$ of the form $Y=-\mathbf{T}_{A(0,-n)}$ with $n \leq 4$. Then, all minimal, strongly convex symplectic fillings of $\left(Y, \xi_{Y}\right)$ have vanishing first Chern class and first and third Betti numbers, and second Betti number equal to $4-n$. Moreover, if $n<4$ they are pairwise orientation-preserving diffeomorphic, while if $n=4$ they fall into at most two diffeomorphism classes.

Proof. Let $P$ be a strongly convex symplectic filling of $\left(Y, \xi_{Y}\right)$. Let $W_{Y}$ be the symplectic cap of Theorem 2.5. Case 3, and let $X$ be the closed, symplectic 4-manifold obtained by gluing $W_{Y}$ 
and $P$ together. The spherical symplectic divisor $D$ is contained in $X$, and therefore $X$ contains a symplectic sphere $F$ having self-intersection 0 and a symplectic sphere $C$ of self-intersection $n$, with $F \cdot C=2$. Thus, according to [16, Theorem 1.4 and Corollary 1.5], $X$ is symplectomorphic to a symplectic blowup of a symplectic $S^{2}$-bundle $p: X_{0} \rightarrow B$, in such a way that $F$ is mapped to a fiber. Let $e_{1}, \ldots, e_{N} \in H_{2}(X ; \mathbb{Z})$ be the exceptional classes. Recall that a basis of the group $H_{2}(X ; \mathbb{Z})$ is given by the classes $[S],[F], e_{1}, \ldots, e_{\ell}$, where $S$ is a section of $p$. Moreover, both $[S]$ and $[F]$ are orthogonal to the classes $e_{i}$ and $[S] \cdot[F]=1$. Therefore we have $[C]=2[S]+a[F]+\sum_{i} x_{i} e_{i}$ for some $a, x_{i} \in \mathbb{Z}$. We now claim that the base $B$ of the fibration has genus $g=0$. In fact, suppose by contradiction that $g>0$. Then, there exist $\alpha, \beta \in H^{1}(B ; \mathbb{R})$ such that $\langle\alpha \cup \beta,[B]\rangle \neq 0$. Viewing $H^{2}\left(X_{0} ; \mathbb{R}\right)$ as the subspace of $H^{2}(X ; \mathbb{R})$ consisting of those classes which vanish on $e_{1}, \ldots, e_{\ell}$, we have

$$
\begin{aligned}
\left\langle p^{*}(\alpha) \cup p^{*}(\beta),[C]\right\rangle & =\left\langle p^{*}(\alpha) \cup p^{*}(\beta), 2[S]+a[F]\right\rangle= \\
& =\left\langle\alpha \cup \beta, p_{*}(2[S]+a[F])\right\rangle=2\langle\alpha \cup \beta,[B]\rangle \neq 0 .
\end{aligned}
$$

On the other hand, since $C$ is a sphere the group $H^{1}(C ; \mathbb{R})$ vanishes, therefore

$$
\left\langle p^{*}(\alpha) \cup p^{*}(\beta),[C]\right\rangle=0 .
$$

This contradiction shows that $g=0$. That is, $X_{0}$ fibers over $\mathbb{C P}^{1}$. Since there are two symplectic fibrations over $\mathbb{C P}^{1}$ up to symplectomorphism, this means that $X$ is either a blowup of $\mathbb{C P}^{2} \# \overline{C P}^{2}$ or a blowup of $S^{2} \times S^{2}$. In the first case $[F]=h-e_{1}$ and $[C]=a h-\sum b_{i} e_{i}$, where $h$ is the class of a line in $\mathbb{C P}^{2}$ and $a>0, b_{i} \geq 0$ by positivity of intersections [17]. In the second case $[F]=f$ and $[C]=a s+b f-\sum c_{i} e_{i}$, where $s=\left[S^{2} \times\{*\}\right], f=\left[\{*\} \times S^{2}\right]$ and by positivity of intersections $a, b, c_{i} \geq 0$. Notice that in both cases we have $b_{1}(X)=0$. Since $b_{1}\left(W_{Y}\right)=b_{1}(Y)=1$, the same Mayer-Vietoris argument used in the proof of Theorem 3.1 shows that $b_{1}(P)=b_{3}(P)=0$. When $n=4$ the statement follows directly from Lemma 3.3 , therefore from now on we assume $n<4$. Our strategy will be to reduce the case $n<4$ to the case $n=4$.

We first analyze the case when $X$ is a blowup of $\mathbb{C P}^{2} \# \overline{\mathbb{C P}}^{2}$. We have three equations satisfied by $n, a$ and the numbers $b_{i}$. The first one comes from the self-intersection of $C$, the second one from the adjunction formula and the third one from the fact that $C$ intersects $F$ twice. They are given, respectively, by:

$$
\left\{\begin{array}{l}
n=a^{2}-\sum b_{i}^{2}, \\
3 a-\sum b_{i}=n+2, \\
a-b_{1}=2
\end{array}\right.
$$

Subtracting the third equation from the second in (3) we obtain

$$
2 a-\sum_{i>1} b_{i}=n
$$

The third equation in (3) implies that $a^{2}-b_{1}^{2}=a^{2}-(a-2)^{2}=4 a-4$. Substituting this into the first equation in (3) we get

$$
4 a-4-\sum_{i>1} b_{i}^{2}=n .
$$

Now we subtract twice Equation (4) from Equation (5), obtaining

$$
\sum_{i>1}\left(2 b_{i}-b_{i}^{2}\right)=4-n \text {. }
$$


Since $n \leq 4$, the sum on the left-hand side must be nonnegative. For each index $i$ we have $2 b_{i}-b_{i}^{2} \leq 1$, and equality holds if and only if $b_{i}=1$, therefore there must be at least $4-n$ indices $i>1$ such that $b_{i}=1$. If there were $m>4-n$ indices $i_{1}, \ldots i_{m}$ such that $b_{i_{1}}=\cdots=b_{i_{m}}=1$, by blowing down the corresponding exceptional spheres we would obtain a configuration $C^{\prime} \cup F$ of symplectic spheres in a closed symplectic 4-manifold contradicting Lemma 3.4. Therefore there must be exactly $4-n$ indices $i>1$ for which $b_{i}=1$. It follows that for all other indices $j$ we have $b_{j} \in\{0,2\}$. However, by positivity of intersections, $b_{j}=0$ corresponds to an exceptional divisors $e_{j}$ disjoint from $C \cup F$, against our assumption of minimality on the filling $P$. On the other hand, if $b_{j}=2$ and $j>1$ then the class $h-e_{1}-e_{j}$ is a represented by an exceptional sphere disjoint both from $C$ and $F$, again contradicting the minimality of $P$.

We conclude that total number of of exceptional classes is exactly $N=5-n$, and that $a=b_{1}+2$ and $b_{2}=\cdots=b_{N}=1$. Substituting these values in the second equation of (3) we obtain $a=2$ and $b_{1}=0$. Summarizing, in this case $X$ is symplectomorphic to $\mathbb{C P}^{2} \#(5-n) \overline{\mathbb{C P}}^{2}$ and the spheres $F$ and $C$ are represented respectively by classes $h-e_{1}$ and $2 h-e_{2}-\cdots-e_{5-n}$. Moreover, from the Mayer-Vietoris sequence we get $b_{2}(P)=4-n$.

Recall that in the second case, i.e. when $X$ is a blowup of $S^{2} \times S^{2}$, we have $[F]=f$ and $[C]=a s+b f-\sum c_{i} e_{i}$, where $s=\left[S^{2} \times\{*\}\right], f=\left[\{*\} \times S^{2}\right]$ and by positivity of intersections $a, b, c_{i} \geq 0$. In fact, the minimality of $P$ implies $c_{i}>0$ for each $i$. Keeping in mind that the canonical class of $S^{2} \times S^{2}$ is Poincare dual to $-2 s-2 f$, the analogues of Equations (3) are

$$
\left\{\begin{array}{l}
n=2 a b-\sum c_{i}^{2}, \\
2 a+2 b-\sum c_{i}=n+2, \\
a=2
\end{array}\right.
$$

Manipulating the equations as in the previous case we obtain

$$
\sum_{i \geq 1}\left(2 c_{i}-c_{i}^{2}\right)=4-n
$$

from which we deduce that $c_{i} \in\{1,2\}$ for each $i$. Finally, we observe that if $c_{i}=2$, the class $f-e_{i}$ is represented by an exceptional sphere disjoint from $C \cup F$, contradicting the minimality of $P$. Therefore $c_{i}=1$ for each $i$ and $b=1$. We conclude that $X$ is symplectomorphic to $\left(S^{2} \times S^{2}\right) \#(4-n) \overline{\mathbb{C P}}^{2}$ and the classes of $F$ of $C$ are given by $f$ and $2 s+f-e_{1}-\cdots-e_{4-n}$ respectively. As before, the Mayer-Vietoris sequence yields $b_{2}(P)=4-n$.

As in the proof of Theorem 3.1, up to isotopy we may assume that $D=F \cup C$ is the strict transform of a configuration $\left(\ell \mathcal{C}_{2}\right)$ or $S^{2} \times\{*\} \cup \Gamma$, each of which carries a homology class Poincaré dual to $c_{1}(X)$. Therefore, in each of the two cases the complement $K$ in $X$ of a regular neighborhood of the configuration $D$ is determined up to diffeomorphisms and the restriction of $c_{1}(X)$ to $K$ vanishes. This implies that the symplectic filling $P$ belongs to one of the two diffeomorphism classes above and that $c_{1}(P)=0$.

In order to finish the proof it suffices to show that if $n<4$ the complements of regular neighborhoods of the configuration in the two cases are diffeomorphic.

Observe that $\mathbb{C P}^{2} \# 2 \overline{\mathbb{C P}}^{2}$ contains an exceptional sphere $R$ representing the characteristic class $h-e_{1}-e_{2}$ and a symplectic sphere $T$ with $[T]=h-e_{1}$ and $T \cap R=\emptyset$. This implies that $\mathbb{C P}^{2} \# 2 \overline{\mathbb{C P}}^{2}$ is a symplectic blowup of a a spin, symplectic 4 -manifold $Z$ containing a symplectic sphere of square zero. By the results of [16], $Z$ is diffeomorphic to $S^{2} \times S^{2}$. This shows that there is a symplectomorphism $\psi: \mathbb{C P}^{2} \# 2 \overline{\mathbb{C P}}^{2} \rightarrow\left(S^{2} \times S^{2}\right) \# \overline{\mathbb{C P}}^{2}$, sending the class $h-e_{1}-e_{2}$ to the exceptional class $e$ and the class $h-e_{1}$ to $f$. It is easy to check that $\psi$ must also send the homology class $2 h-e_{2}$ to the homology class $2 s+f-e$. Gromov's results [9] imply that, up to isotopy, $\psi$ maps the strict transform of a line representing the class $h-e_{1}$ to the strict trasforn of a sphere 
$\{*\} \times S^{2}$ representing the class $f$, and the strict transform of a conic representing the class $2 h-e_{2}$ to the strict transform of a graph $\Gamma$ representing the homology class $2 s+f-e$. Clearly, for each $m \geq 2$ there is a symplectomorphism between $\mathbb{C P}^{2} \# m \overline{\mathbb{C P}}^{2}$ and $S^{2} \times S^{2} \#(m-1) \overline{\mathbb{C P}}^{2}$ with the same properties. This shows that the complements of regular neighborhoods of the configuration in the two cases are diffeomorphic to each other, and concludes the proof.

In view of Theorem 3.1, it is natural to wonder how many diffeomorphism types of strongly convex, minimal symplectic fillings a given contact hyperbolic torus bundle $\left(Y, \xi_{Y}\right)$ may have. We do not answer this question in general, but we are able to establish the following result.

Proposition 3.6. There exist infinitely many contact hyperbolic torus bundles $\left(Y, \xi_{Y}\right)$ admitting non-homotopy equivalent Stein fillings.

Proof. Let us denote by $\Gamma\left(1,1-c_{1},-c_{2}, \ldots,-c_{\ell-1}, 1-c_{\ell}\right)$ the graph on the left of Figure 5 Inside a blowup $\mathbb{C P}^{2} \# \overline{\mathbb{C P}^{2}}$ of the standard Kähler $\mathbb{C P}^{2}$ we can easily find a spherical complex divisor $D(1,0,-1,0)$ having dual intersection graph $\Gamma(1,0,-1,0)$ and whose complex spheres represent homology classes $h, h-e_{1}, e_{1}$ and $h-e_{1}$, where $h$ is the hyperplane class and $e_{1}$ is the exceptional class. Blowing up at the appropriate nodal point of the divisor and taking its proper trasform we get a spherical complex divisor $D(1,-1,-1,-2,0)$ inside $\mathbb{C P}^{2} \# 2 \overline{\mathbb{C P}^{2}}$ with dual intersection graph $\Gamma(1,-1,-1,-2,0)$. Blowing up again we get a divisor $D(1,-1,-2,-1,-3,0)$ inside $\mathbb{C P}^{2} \# 3 \overline{\mathbb{C P}^{2}}$, whose spheres represent the classes $h, h-e_{1}-e_{2}, e_{2}-e_{3}, e_{3}, e_{1}-e_{2}-e_{3}$ and $h-e_{1}$, where the classes $e_{i}$ are the exceptional classes. Now we blow up in two different ways. First we blow up in such a way as to obtain a divisor $D_{1}=D(1,-2,-1,-3,-1,-3,0) \subset \mathbb{C P}^{2} \# 4 \overline{\mathbb{C P}^{2}}$, with spheres representing the classes:

$$
h, \quad h-e_{1}-e_{2}-e_{4}, \quad e_{4}, \quad e_{2}-e_{3}-e_{4}, \quad e_{3}, \quad e_{1}-e_{2}-e_{3} \quad \text { and } h-e_{1} .
$$

Then, we blow up so as to obtain a divisor $D_{2}=D(1,-1,-3,-1,-2,-3,0) \subset \mathbb{C} \mathbb{P}^{2} \# 4 \overline{\mathbb{C P}^{2}}$, with spheres representing the classes:

$$
h, \quad h-e_{1}-e_{2}, \quad e_{2}-e_{3}-e_{4}, \quad e_{4}, \quad e_{3}-e_{4}, \quad e_{1}-e_{2}-e_{3} \quad \text { and } h-e_{1} .
$$

Finally, we suitably blow up another five times at smooth points of both $D_{1}$ and $D_{2}$, so that upon taking proper transforms we obtain two distinct divisors $D_{1}^{(0)}$ and $D_{2}^{(0)}$ inside $\mathbb{C P}^{2} \# 9 \overline{\mathbb{C P}^{2}}$ with the same dual intersection graph $\Gamma(1,-2,-3,-3,-2,-3,-2)$.

Let $P_{i}^{(0)}$, for $i=1,2$, denote the closure of a regular neighborhood $W_{i}^{(0)}$ of $D_{i}^{(0)}$. We claim that $P_{1}^{(0)}$ and $P_{2}^{(0)}$ are not homotopy equivalent. For the rest of this proof, whenever we mention homology groups we shall always implicitly use integer coefficients. Using the definition of $W_{i}^{(0)}$ one can check that the second homology group $H_{2}\left(W_{i}^{(0)}\right)$ is free of rank 7. Let $X$ be the complex projective plane blown up nine times. Using the Mayer-Vietoris sequence for the decomposition $X=W_{i}^{(0)} \cup P_{i}^{(0)}$ one can check that $H_{2}\left(P_{i}^{(0)}\right)$ is free Abelian of rank 4, and that its image $j_{*}\left(H_{2}\left(P_{i}^{(0)}\right)\right)$ under the map induced by the inclusion $j: P_{i}^{(0)} \rightarrow X$ is isometric, as an intersection lattice, to $H_{2}\left(P_{i}^{(0)}\right) /\langle T\rangle$, where $\langle T\rangle$ denotes the free, rank-1 subgroup generated by the class of the torus fiber in the boundary (pushed in the interior), which coincides with the kernel of the intersection pairing on $H_{2}\left(P_{i}^{(0)}\right)$. This implies that the isometry class of the intersection lattice $j_{*}\left(H_{2}\left(P_{i}^{(0)}\right)\right)$ is determined by the homotopy type of $P_{i}^{(0)}$. We claim that $j_{*}\left(H_{2}\left(P_{1}^{(0)}\right)\right)$ and $j_{*}\left(H_{2}\left(P_{2}^{(0)}\right)\right)$ are not isometric to each other, which in turn implies that $P_{1}^{(0)}$ and $P_{2}^{(0)}$ are not homotopy equivalent. To prove the claim we shall use the fact that, for $i=1,2, j_{*}\left(H_{2}\left(P_{i}^{(0)}\right)\right)$ is isometric to the lattice $\Lambda_{i}^{(0)}$ orthogonal to the image of $H_{2}\left(W_{i}\right)$ 
under the map induced by the inclusion $W_{i} \subset X$. To see this fact one may observe that, given any $a \in H_{2}(X)$ orthogonal to all the homology classes of the spheres belonging to the divisor $D_{i} \subset W_{i}$, one can represent $a$ by a smooth, oriented surface disjoint from $D_{i}$, and therefore $a \in j_{*}\left(H_{2}\left(P_{i}\right)\right)$.

We now set out to compute the determinant of $\Lambda_{i}^{(0)}$. The classes of the spheres of $D_{1}^{(0)}$ are

$$
h, h-e_{1}-e_{2}-e_{4}, e_{4}-e_{5}-e_{6}, e_{2}-e_{3}-e_{4}, e_{3}-e_{7}, e_{1}-e_{2}-e_{3}, h-e_{1}-e_{8}-e_{9},
$$

while the classes of the spheres of $D_{2}^{(0)}$ are

$$
h, h-e_{1}-e_{2}-e_{5}, e_{2}-e_{3}-e_{4}, e_{4}-e_{6}-e_{7}, e_{3}-e_{4}, e_{1}-e_{2}-e_{3}, h-e_{1}-e_{8}-e_{9} .
$$

A direct calculation shows that the sublattice $\Lambda_{1}^{(0)}$ of $H_{2}\left(\mathbb{C P}^{2} \# 9 \overline{\mathbb{C P}^{2}}\right)$ orthogonal to the classes of $D_{1}^{(0)}$ has integral basis:

$$
\alpha_{1}=e_{5}-e_{6}, \quad \alpha_{2}=e_{1}+e_{3}-e_{4}-e_{5}+e_{7}-e_{8}, \quad \alpha_{3}=e_{8}-e_{9} .
$$

On the other hand, the sublattice $\Lambda_{2}^{(0)}$ orthogonal to the classes of $D_{1}^{(0)}$ has integral basis:

$$
\beta_{1}=e_{6}-e_{7}, \quad \beta_{2}=-3 e_{1}-2 e_{2}-e_{3}-e_{4}+5 e_{5}-e_{6}+3 e_{9}, \quad \beta_{3}=e_{8}-e_{9} .
$$

This shows that the lattices $\Lambda_{1}^{(0)}$ and $\Lambda_{2}^{(0)}$ are both even. The intersection matrix $\left(\alpha_{i} \cdot \alpha_{j}\right)$ has determinant -20 , while the intersection matrix $\left(\beta_{i} \cdot \beta_{j}\right)$ has determinant -180 , therefore $\Lambda_{1}$ and $\Lambda_{2}$ are not isometric, and $P_{1}^{(0)}$ and $P_{2}^{(0)}$ are not homotopy equivalent. Moreover, applying Theorem 2.1 and the results of [2] as in the proof of Theorem 2.5 shows that $P_{1}^{(0)}$ and $P_{2}^{(0)}$ can be endowed with structures of Stein fillings of $\left(-\mathbf{T}_{-A(3,3,3,2,3,3)}, \xi_{\left.-\mathbf{T}_{-A(3,3,3,2,3,3)}\right)}\right.$.

This example belongs to an infinite family of examples obtained as follows. We blow up at $N \geq 1$ generic points of the sphere of $D_{1}^{(0)}$ representing $e_{2}-e_{3}-e_{4}$, and at $N$ generic points of the sphere of $D_{2}^{(0)}$ representing $e_{4}-e_{6}-e_{7}$. Taking proper transforms we get spherical complex divisors $D_{i}^{(N)} \subset \mathbb{C P}^{2} \#(N+9) \overline{\mathbb{C P}^{2}}$ having dual intersection graphs $\Gamma(-1,-2,-3,-3-N,-2,-3,-2)$ and determining Stein fillings $P_{i}^{(N)}, i=1,2$. Arguing as for $D_{i}^{(0)}$, we get that the orthogonal lattice $\Lambda_{1}^{(N)}$ has integral basis

$$
\alpha_{1}, \quad \alpha_{2}, \quad \alpha_{3}, \quad \alpha_{4}=e_{9}-e_{10}, \quad \alpha_{5}=e_{10}-e_{11}, \quad \ldots \quad \alpha_{N+6}=e_{N+8}-e_{N+9},
$$

while $\Lambda_{2}^{(N)}$ has integral basis

$$
\beta_{1}, \quad \beta_{2}, \quad \beta_{3}, \quad \beta_{4}=e_{9}-e_{10}, \quad \beta_{5}=e_{10}-e_{11}, \quad \ldots \quad \beta_{N+6}=e_{N+8}-e_{N+9} .
$$

Then, an inductive computation yields

$$
\operatorname{det}\left(\alpha_{i} \cdot \alpha_{j}\right)=(-1)^{N+1}(9 N+20) \text { and } \operatorname{det}\left(\beta_{i} \cdot \beta_{j}\right)=(-1)^{N+1} 9(9 N+20) .
$$

This shows that $P_{1}^{(N)}$ and $P_{2}^{(N)}$ are non-homotopy equivalent and carry structures of Stein fillings of the same contact hyperbolic bundle for each $N \geq 0$.

\section{IDENTIFYING THE CONTACT STRUCTURES}

In this section we use Honda's classification [10] of tight contact structures on torus bundles over the circle (see also [7]) to identify the contact structures $\xi_{Y}$ for elliptic bundles of the form $Y=\mathbf{T}_{-A(\varepsilon)}$, with $\varepsilon \in\{-1,0,1\}$ and $Y=\mathbf{T}_{A(1)}$, as well as for the hyperbolic bundles of Theorem 1.1.4). We also give explicit constructions of Stein fillings for $\left(Y, \xi_{Y}\right)$ when $Y$ is elliptic as above. 
Proposition 4.1. Let $Y$ be an elliptic torus bundle of the form $Y=\mathbf{T}_{-A(\varepsilon)}$, with $\varepsilon \in\{-1,0,1\}$ or $Y=\mathbf{T}_{A(1)}$. Then, the contact structure $\xi_{Y}$ is the unique tight contact structure on $Y$ with vanishing Giroux torsion. Moreover, $\xi_{Y}$ is universally tight.

Proof. The bundles in question are associated to the monodromies

$$
-A(-1)=\left(\begin{array}{cc}
1 & -1 \\
1 & 0
\end{array}\right), \quad-A(0)=\left(\begin{array}{cc}
0 & -1 \\
1 & 0
\end{array}\right), \quad-A(1)=\left(\begin{array}{cc}
-1 & -1 \\
1 & 0
\end{array}\right) \quad \text { and } \quad A(1)=\left(\begin{array}{cc}
1 & 1 \\
-1 & 0
\end{array}\right) .
$$

Defining $S=A(0)$ and $T=\left(\begin{array}{ll}1 & 1 \\ 0 & 1\end{array}\right)$, it is easy to check that the first monodromy is conjugate to $-\left(T^{-1} S\right)^{2}$, the second and third ones are equal, respectively, to $-S$ and $-T^{-1} S$ and the last one to $T^{-1} S$. Then, Honda's classification [10] implies that on the associated bundles there is only one isotopy class of tight contact structures without Giroux torsion, and that this isotopy class is universally tight (there are no virtually overtwisted contact structures on these bundles). Since fillable contact structures have no Giroux torsion [5], the contact structure $\xi_{Y}$ must be isotopic to the unique tight contact structure on $Y$ without Giroux torsion.

It might be interesting to see an explicit construction of a Stein filling of $\left(Y, \xi_{Y}\right)$ for the bundles of Proposition 4.1. It follows from the proposition and the fact that fillable contact structures have no Giroux torsion [5] that $\xi_{Y}$ is the unique Stein fillable contact structure on $Y$. Therefore, in order to exhibit a Stein filling of $\left(Y, \xi_{Y}\right)$ it suffices to construct a single Stein 4-manifold with boundary $X$ such that $\partial X=Y$. Starting from the obvious Kirby diagrams corresponding to the graphs of Figures 2 and 3 and using Kirby calculus it is a simple matter to check that each of the torus bundles $\mathbf{T}_{-A(\varepsilon)}, \varepsilon \in\{-1,0,1\}$, is the boundary of the 4-dimensional plumbing given in Figure 6 . In the

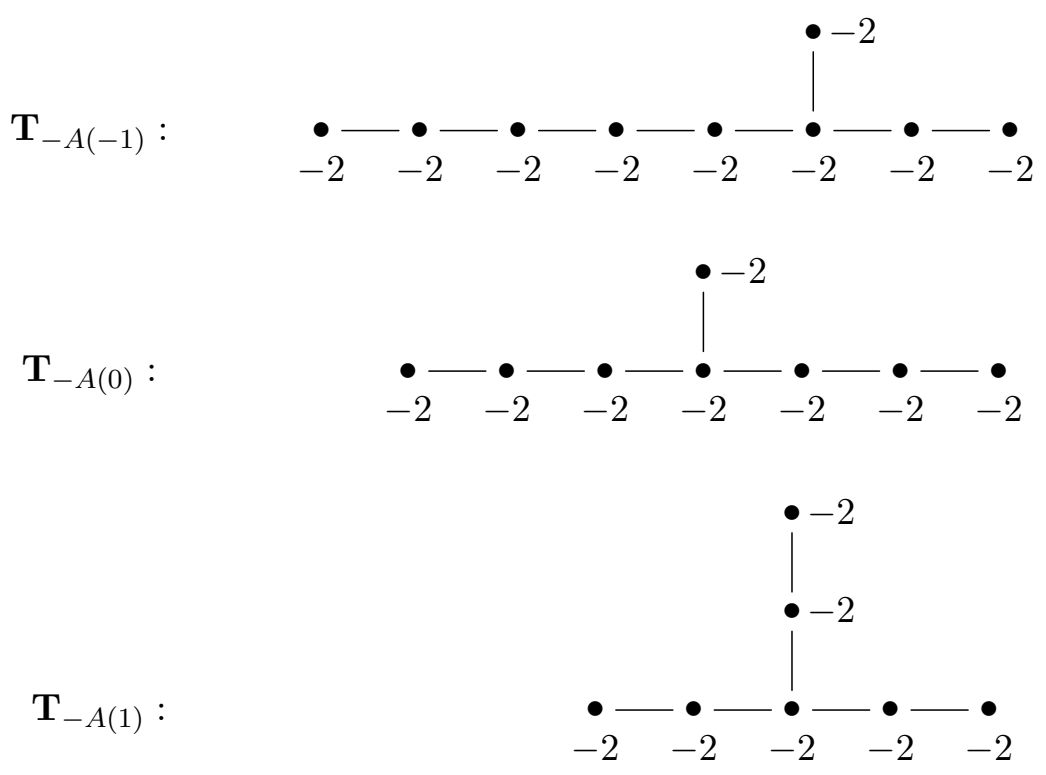

FIGURE 6. Plumbings bounding the elliptic bundles $\mathbf{T}_{-A(\varepsilon)}, \varepsilon \in\{-1,0,1\}$.

same way it is easy to check that the bundle $\mathbf{T}_{A(1)}$ is the boundary of the smooth 4-dimensional handlebody obtained by attaching a 4-dimensional 2-handle to the 4-ball along the right-handed trefoil knot in $S^{3}$ with framing 0. Using e.g. the results of [8] it is straightforward to check that each one of the smooth 4-manifolds just described carries a Stein structure with boundary and therefore gives a Stein filling of the corresponding torus bundle. 
We now consider hyperbolic bundles. Let $Y$ by a hyperbolic torus bundle with $Y=\mathbf{T}_{A}$ and $\operatorname{tr}(A)<-2$. Then, as explained in Section 1, $Y=\mathbf{T}_{-A(d)}$, where $d=\left(d_{m}, d_{m-1}, \ldots, d_{1}\right), d_{i} \geq 2$ for all $i$ and $d_{i} \geq 3$ for some $i$. Moreover, by [19. Theorem 6.1] $Y$ is the oriented boundary of the 4-dimensional plumbing $P_{-}(d)$ given by the diagram of Figure 7

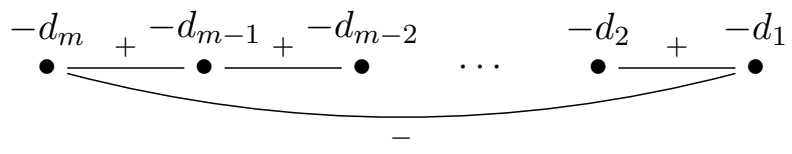

Figure 7. Plumbings $P_{-}(d)$ bounding the hyperbolic bundles $\mathbf{T}_{-A(d)}$.

Lemma $4.2\left([7,[10])\right.$. $\mathbf{T}_{-A(d)}$ carries $\left(d_{1}-1\right)\left(d_{2}-1\right) \cdots\left(d_{m}-1\right)$ tight, virtually overtwisted contact structures up to isotopy and one universally tight contact structure with no Giroux torsion up to contactomorphisms.

Proof. One can easily check that $-A(d)=-T^{-d_{1}} S T^{-d_{2}} S \cdots T^{-d_{m-1}} S T^{-d_{m}} S$, where $S=A(0)$ and $T=\left(\begin{array}{ll}1 & 1 \\ 0 & 1\end{array}\right)$. The results of [7, 10] are written in terms of such a factorization of $-A(d)$, and they immediately imply the statement (see e.g. the table on page 90 of [10]).

Lemma 4.3. Let $Y$ by a hyperbolic torus bundle with $Y=\mathbf{T}_{-A(d)}$. Then, each virtually overtwisted tight contact structure on $Y$ admits a Stein filling $P$ with $b_{1}(P)=1$.

Proof. Figure 8 represents the front diagram of a Legendrian link $\mathbb{L}$ inside the standard contact $S^{2} \times S^{1}$ viewed as $S^{3}$ with a 3-dimensional 1-handle attached (see e.g. [8]). Recall that there is no need to specify the over/under information at each crossing of the diagram, because the over-strand is always the one with smaller slope. Label the components of the link $\mathbb{L}$ as $L_{1}, \ldots, L_{m}$ from left to right, with $L_{m}$ being the component going over the 1-handle. Orient the components so that $\operatorname{lk}\left(L_{m}, L_{1}\right)=-1$ and $\operatorname{lk}\left(L_{j}, L_{j+1}\right)=1$ for any $1 \leq j<m$, and Legendrian stabilize $d_{j}-2$ times each component. For each index $j, L_{j}$ becomes a Legendrian unknot (which we keep denoting $L_{j}$ ) with $\operatorname{tb}\left(L_{j}\right)=-d_{j}+1$. Since there are $d_{j}-1$ ways to stabilize an oriented Legendrian knot $d_{j}-2$ times, for each $j$ we get $d_{j}-1$ isotopy classes of such unknots, distinguished by their rotation numbers. We can then attach a 4-dimensional Stein handle along each $L_{j}$ to $S^{2} \times S^{1}$ viewed as the boundary of $B^{4}$ union a 4-dimensional 1-handle, obtaining $\prod_{j}\left(d_{j}-1\right)$ Stein structures with boundary on the smooth 4-dimensional plumbing $P_{-}(d)$. Notice that $\pi_{1}\left(P_{-}(d)\right)=\mathbb{Z}$, and in particular $b_{1}\left(P_{-}(d)\right)=1$. We now want to argue that the $\prod_{j}\left(d_{j}-1\right)$ Stein 4 -manifolds we just

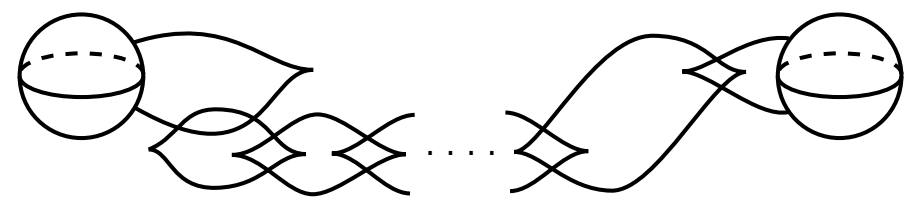

FIGURE 8. The Legendrian link $\mathbb{L}$

described yield distinct isotopy classes of contact structures on $\partial P_{-}(d)=\mathbf{T}_{-A(d)}$. Fix a choice of stabilizations, or equivalently the $m$-tuple $r=\left(r_{1}, \ldots, r_{m}\right)=\left(\operatorname{rot}\left(L_{1}\right), \ldots, \operatorname{rot}\left(L_{m}\right)\right)$ of rotation numbers of the components of $\mathbb{L}$, and let $J_{r}, \xi_{r}$ be the associated Stein structure on $P_{-}(d)$ and induced contact structure on $\mathbf{T}_{-A(d)}$, respectively. Also, let $S_{j}$ be the homology class carried by the oriented $j$-th sphere of the plumbing obtained by capping off an oriented disk bounding $L_{j}$ with 
the core of the attached 4-dimensional 2-handle. Since $\left\langle c_{1}\left(J_{r}\right), S_{j}\right\rangle=r_{j}$, by [14, Theorem 1.2] the contact structures $\xi_{r}, \xi_{r^{\prime}}$ corresponding to the two $m$-tuples $r, r^{\prime}$ can be isotopic only if $r=r^{\prime}$. Thus, the collection $\left\{\xi_{r}\right\}_{r}$ consists of $\left(d_{1}-1\right)\left(d_{2}-1\right) \cdots\left(d_{m}-1\right)$ pairwise nonisotopic contact structures on $\mathbf{T}_{-A(d)}$, each admitting a Stein filling $P$ with $b_{1}(P)=1$. In view of Lemma 4.2 , in order to finish the proof it suffices to show that $\xi_{r}$ is virtually overtwisted for every $r$. Consider the double cover $\widetilde{P_{-}(d)} \rightarrow P_{-}(d)$ associated to the subgroup $2 \mathbb{Z} \subset \mathbb{Z}=\pi_{1}\left(P_{-}(d)\right)$. If we denote with $d^{\prime}$ the string $(d, d)$, it is easy to check that $\widetilde{P_{-}(d)}=P_{+}\left(d^{\prime}\right)$, where $P_{+}\left(d^{\prime}\right)$ is the plumbing described by the diagram of Figure 9 . Moreover, by the proof of [19. Theorem 6.1] we have $\partial P_{+}\left(d^{\prime}\right)=\mathbf{T}_{A\left(d^{\prime}\right)}$.

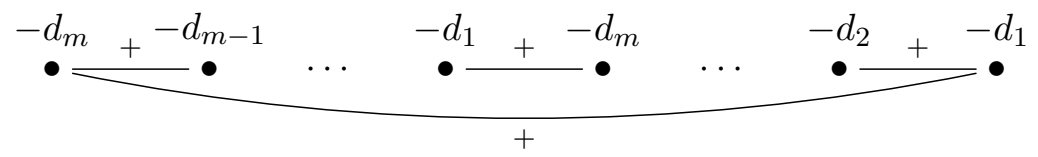

FIGURE 9. The 4-dimensional plumbing $P_{+}\left(d^{\prime}\right)$.

The Stein structure $J_{r}$ on $P_{-}(d)$ pulls back to a Stein structure $\widetilde{J}_{r}$ on $\widetilde{P_{-}(d)}$, obtained by attaching Stein handles along suitably oriented and stabilized components of the Legendrian link $\mathbb{L}^{\prime}=$ $L_{1}^{\prime} \cup \cdots \cup L_{2 m}^{\prime}$ of Figure 10 . With the orientations and stabilizations just described, $L_{i}^{\prime}$ and $L_{i+m}^{\prime}$

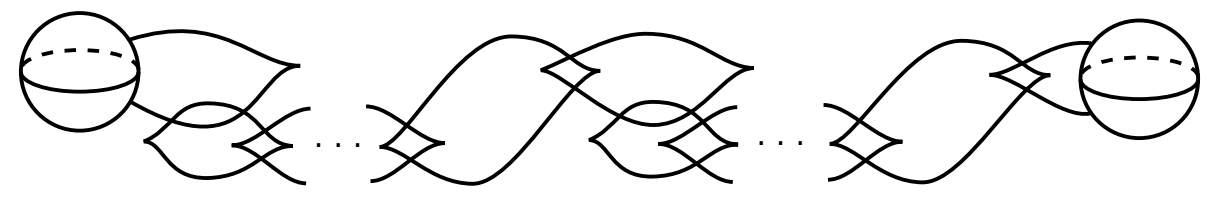

FIGURE 10. The Legendrian link $\mathbb{L}^{\prime}$

acquire the same rotation number $r_{i}$ for all $i$ (where it is understood that $L_{2 m+1}^{\prime}=L_{1}^{\prime}$ ).

We want to compare the Stein structures $J_{r}$ on $P_{+}\left(d^{\prime}\right)$ with the Stein structures constructed by Bhupal and Ozbagci on the same 4-manifold [1]. Let $S_{j}^{\prime} \in H_{2}\left(P_{+}\left(d^{\prime}\right)\right), j=1, \ldots, m$, be the homology class carried by the oriented spheres obtained by capping off an oriented disk bounding $L_{j}^{\prime}$ with the core of the 4-dimensional 2-handle. Observe that $S_{j}^{\prime} \cdot S_{j+1}^{\prime}=+1$ for $j=1, \ldots, m-1$. Now we choose further homology classes $S_{j}^{\prime} \in H_{2}\left(P_{+}\left(d^{\prime}\right)\right), j=m+1, \ldots, 2 m$, carried by spheres obtained by capping off disks as before, but we orient the spheres so that $S_{j}^{\prime} \cdot S_{j+1}^{\prime}=1$ for every $i$ (where it is understood that $S_{2 m+1}^{\prime}=S_{1}^{\prime}$ ). This implies that the classes $S_{m+1}^{\prime}, \ldots, S_{2 m}^{\prime}$ are carried by spheres obtained by capping off oriented discs bounding $-L_{m+1}^{\prime}, \ldots,-L_{2 m}^{\prime}$. Therefore $\left\langle c_{1}\left(\tilde{J}_{r}\right), S_{j}^{\prime}\right\rangle=r_{j}$ if $1 \leq j \leq m$, while $\left\langle c_{1}\left(\tilde{J}_{r}\right), S_{j}\right\rangle=-r_{j}$ for $m<j \leq 2 m$. On the other hand, according to [1, Proposition 11] for each universally tight contact structure on $\partial P_{+}\left(d^{\prime}\right)=\mathbf{T}_{A\left(d^{\prime}\right)}$ there is a Stein structure $J$ on $P_{+}\left(d^{\prime}\right)$ such that $\left\langle c_{1}(J), S_{j}^{\prime}\right\rangle=\varepsilon\left(d_{j}-2\right)$ for each $j$, where $\varepsilon= \pm 1$ is independent of $j$ (to check the orientations of the spheres see [1, Figure 6]). Applying [14] we conclude that, in order for the contact structure $\tilde{\xi}_{r}$ induced on $\mathbf{T}_{A\left(d^{\prime}\right)}$ to be universally tight, we would need to have $d_{j}=2$ for each $j$. But this contradicts our assumption that $d_{j} \geq 3$ for at least one $j$. Therefore, each $\tilde{\xi}_{r}$ is virtually overtwisted, and so is $\xi_{r}$. This concludes the proof.

We are now ready to state our result for hyperbolic bundles. 
Theorem 4.4. Let $Y$ by a hyperbolic torus bundle with $Y=\mathbf{T}_{-A(d)}$ with dembeddable. Then, the contact structure $\xi_{Y}$ is the unique universally tight contact structure on $Y$ with vanishing Giroux torsion.

Proof. By Theorem 2.5 the contact structure $\xi_{Y}$ is Stein fillable, and by [5] it has no Giroux torsion. Suppose that $\xi_{Y}$ is virtually overtwisted. Then, by Lemma 4.3, $\left(Y, \xi_{Y}\right)$ admits a Stein filling $P$ with $b_{1}(P)=1$. But by Theorem 3.1 each Stein filling of $\left(Y, \xi_{Y}\right)$ has vanishing first Betti number. This shows that $\xi_{Y}$ is universally tight, and Honda's classification [10] implies that on the underlying bundle there is only one isotopy class of universally tight contact structures without Giroux torsion (see e.g. the table of [10, Page 90]).

\section{REFERENCES}

[1] Mohan Bhupal and Burak Ozbagci, Canonical contact structures on some singularity links, Bull. London Math. Soc. 46 (2014), no. 3, 576-586.

[2] Fedor A Bogomolov and Bruno de Oliveira, Stein small deformations of strictly pseudoconvex surfaces, Contemp. Math. 207 (1997), 25-42.

[3] Yakov Eliashberg, Filling by holomorphic discs and its applications, Geometry of low-dimensional manifolds, 2 (Durham, 1989), 1990, pp. 45-67.

[4] Sandra Francisco, Symplectic isotopy for cuspidal curves, Ph.D. Thesis, Massachusetts Institute of Technology, 2005.

[5] David T Gay, Four-dimensional symplectic cobordisms containing three-handles, Geom. Topol. 10 (2006), 1749-1759.

[6] Hansjörg Geiges, An introduction to contact topology, Cambridge Univ. Press, 2008.

[7] Emmanuel Giroux, Structures de contact en dimension trois et bifurcations des feuilletages de surfaces, Invent. Math. 141 (2000), no. 3, 615-689.

[8] Robert E Gompf, Handlebody construction of Stein surfaces, Ann. Math. (1998), 619-693.

[9] Mikhael Gromov, Pseudo holomorphic curves in symplectic manifolds, Invent. Math. 82 (1985), no. 2, $307-347$.

[10] Ko Honda et al., On the classification of tight contact structures II, J. Diff. Geom. 55 (2000), no. 1, 83-143.

[11] Tian-Jun Li and Cheuk Yu Mak, Symplectic divisorial capping in dimension 4, arXiv:1407.0564 (2014).

[12] Paolo Lisca, On symplectic fillings of lens spaces, Trans. Amer. Math. Soc. 360 (2008), no. 2, 765-799.

[13] _ Stein fillable contact 3-manifolds and positive open books of genus one, Algebr. Geom. Topol. 14 (2014), no. 4, 531-550.

[14] Paolo Lisca and Gordana Matić, Tight contact structures and Seiberg-Witten invariants, Invent. Math. 129 (1997), no. 3, 509-525.

[15] Ai-Ko Liu, Some new applications of general wall crossing formula, Gompf's conjecture and its applications, Math. Res. Lett. 3 (1996), 569-586.

[16] Dusa McDuff, The structure of rational and ruled symplectic 4-manifolds, J. Amer. Math. Soc. 3 (1990), no. 3 , 679-712.

[17] _ The local behaviour of holomorphic curves in almost complex 4-manifolds, Journal of Differential Geometry 34 (1991), no. 1, 143-164.

[18] Dusa McDuff and Dietmar Salamon, Introduction to symplectic topology, Second Edition, Oxford Mathematical Monographs, The Clarendon Press, Oxford University Press, New York, 1998.

[19] Walter D Neumann, A calculus for plumbing applied to the topology of complex surface singularities and degenerating complex curves, Trans. Amer. Math. Soc. 268 (1981), no. 2, 299-344.

[20] Hiroshi Ohta and Kaoru Ono, Symplectic fillings of the link of simple elliptic singularities, J. Reine Angew. Math. 565 (2003), 183-205.

[21] _ Simple singularities and symplectic fillings, J. Diff. Geom. 69 (2005), no. 1, 1-42.

[22] Olga Plamenevskaya and Jeremy Van Horn-Morris, Planar open books, monodromy factorizations and symplectic fillings, Geom. Topol. 14 (2010), no. 4, 2077-2101.

[23] Laura Starkston, Symplectic fillings of Seifert fibered spaces, Trans. Amer. Math. Soc. 367 (2015), no. 8, 5971-6016.

Dipartimento di Matematica, Università di Pisa, LARgo Bruno Pontecorvo 5, 56121 Pisa, ItAly

E-mail address: marco.golla@for.unipi.it, lisca@dm.unipi.it 